\title{
Olfactory function in patients with obstructive sleep apnea and the effect of positive airway pressure treatment: a systematic review and meta-analysis
}

\author{
Murat Binar $^{1}$ (D) Mert Cemal Gokgoz ${ }^{2}$ \\ Received: 12 November 2020 / Revised: 26 February 2021 / Accepted: 9 March 2021 / Published online: 18 March 2021 \\ (C) The Author(s), under exclusive licence to Springer Nature Switzerland AG 2021
}

\begin{abstract}
Objectives To investigate olfactory function (OF) in patients with obstructive sleep apnea (OSA) and evaluate whether or not the use of positive airway pressure (PAP) treatment has an impact on olfactory performance.

Methods All studies published in English that gave satisfactory data regarding the assessment of OF in patients with OSA were included in this review. First, a baseline assessment of OF in patients with OSA who had not received any treatment was examined. Second, the effect of PAP therapy on OF was assessed to be able to make before and after comparisons. The primary outcome of this study was the threshold-discrimination identification (TDI) scores, obtained from the Sniffin' Sticks test.

Results The database search identified 552 articles. According to the exclusion criteria, 11 studies involving 557 patients diagnosed with OSA were included in this meta-analysis. The general rate of olfactory dysfunction was $73 \%$ (95\% CI: 56.481-87.057) among the patients with OSA. The patients with OSA had lower TDI scores compared to the control group and the difference was statistically significant $(p<0.001)$. PAP treatment significantly improved the TDI scores in patients with OSA $(p<0.001)$. There was a significant negative correlation between the severity of apnea-hypopnea index and TDI scores $(p=$ $0.001, z=-3.377, r=-0.438)$ and between age and TDI scores $(p=0.007, z=-2.695, r=-0.236)$.

Conclusion This meta-analysis demonstrates that OSA impairs OF, while PAP treatment can reverse the olfactory performance of patients with OSA.
\end{abstract}

Keywords Obstructive sleep apnea $\cdot$ Olfactory $\cdot$ Sniffin' Sticks test $\cdot$ Positive airway pressure $\cdot$ Meta-analysis

\section{Introduction}

Obstructive sleep apnea (OSA) affects millions of people worldwide. OSA is not only an upper airway disease but also a systemic disorder that is associated with many comorbidities such as hypertension, coronary arterial disease, stroke, and metabolic syndrome. Repetitive episodes of partial or complete upper airway collapse during sleep are the main characteristics of OSA [1]. The upper airway, which is the origin of

Murat Binar

mbinar4@yahoo.com

1 Department of Otolaryngology, Head and Neck Surgery, Ento KBB Medical Center, Kazımdirik Mah. 364/1. Sk. No: 36/A,

35100 Bornova, Izmir, Turkey

2 Department of Otolaryngology, Head and Neck Surgery, Manisa City Hospital, Manisa, Turkey
OSA pathophysiology, is also the region where the olfactory pathways begin. Beyond this close regional relationship, the physiological, neurological, and systemic dimensions of these two important subspecialities of otolaryngology raise the need for research on this subject in terms of possible interaction between olfactory function (OF) and OSA.

The sense of smell is one of the most ancient senses of human beings, which directly impacts quality of life, affects interpersonal relationships, and provides protection from dangerous conditions in the environment such as gasses, fire, burns, and rotten food. Patients with olfactory dysfunction may experience restrictions in their daily life activities [2]. Since the loss of smell also leads to taste disorders, the importance of smell functions in social life increases even more. Decreased flavor perception and impaired enjoyment of food may result in reduced appetite which can lead weight gain or weight loss in line with eating more or less [3]. Patients with olfactory dysfunction may be unable to work in some jobs requiring good $\mathrm{OF}$, and impaired ability to perceive one's 
own body odors may lead to self-confidence problems. Furthermore, those patients reportedly have a higher prevalence of depression than is found in the general population [4].

Upper airway infections, sinonasal pathologies, nasal obstruction, tumors, traumas, disorders of the central nervous system, and drugs are common causes of olfactory dysfunction. The impact of COVID-19 must also be mentioned as the most recently reported reason for smell dysfunction [5]. Recent studies have provided consistent evidence regarding the negative effect of OSA on olfactory performance [6-8]. The local effect of OSA on the nasal mucosa, such as longstanding vibrations and local neuropathy, is one of the suggested hypotheses for olfactory dysfunction [7]. Another hypothesis is explained by the neurocognitive effects of OSA that patients with OSA can show impaired cognitive functions depending on sleep fragmentation, intermittent hypoxia, and hypoxia-reoxygenation episodes [8]. However, the exact mechanism leading to olfactory dysfunction in OSA is still unknown.

Although current literature is still poor in terms of understanding OF in patients with OSA, the number of published articles performed using the Sniffin' Sticks test is sufficient to produce a general outcome. This meta-analysis study has two purposes: to investigate OF in patients with OSA and evaluate whether or not positive airway pressure (PAP) treatment has an impact on olfactory performance.

\section{Data and methods}

\section{Literature research}

The Preferred Reporting Items for Systematic Reviews and Meta-analyses statement was followed during this review (https://www.prisma-statement.org). The literature search was performed using MEDLINE, Google Scholar, Cochrane, and SAGE databases. Various combinations of the following search terms were used: snoring, sleep apnea, obstructive sleep apnea, olfactory, olfaction, smell, odor, Sniffin' Sticks, and positive airway pressure. The final update of the review was done on June 1, 2020. The entire literature was reviewed from this date back, and the articles included in this meta-analysis were published between 2014 and 2020.

\section{Inclusion criteria}

All studies published in English that focused on OF assessment in patients with OSA were included in this review. The Sniffin' Sticks test for olfactory measurements and polysomnography for the diagnosis of OSA were the sine qua non of the meta-analysis.

\section{Exclusion criteria}

Studies that focused on patients with a history of upper airway surgery (including septoplasty, rhinoplasty, turbinoplasty, concha ablation, endoscopic sinus surgery, OSA surgeries), upper respiratory infections, sinonasal diseases (including chronic rhinosinusitis, nasal polyps, inferior turbinate hypertrophy, allergic rhinitis), tumors, history of severe head trauma, neurological and psychiatric disorders, and endocrine and metabolic disorders were excluded from this review. Some other articles were also excluded for the following reasons: no smell test, different olfactory test, no description of statistical data, other diseases/treatments, use of a language other than English, duplication of published literature, review/metaanalysis, non-original studies (letters, editorial, comments, posters, chapter, etc.), genetic studies, biochemical studies, animal studies, radiological studies (without smell test), and pediatric studies (Fig. 1).

\section{Data extraction}

The titles and abstracts of the studies were inspected, and data were extracted according to the inclusion/exclusion criteria. These data included the author names, the publication year, the number of reported patients, the average age, gender information, the mean apnea-hypopnea index (AHI), evaluation method for OF, the mean overall threshold-discrimination identification (TDI) scores, the percentage of olfactory dysfunction in patients with OSA, use of PAP treatment, and the outcomes/comments of the studies.

\section{Main outcome measurements}

The Sniffin' Sticks test is a test of nasal chemosensory performance based on pen-like odor dispensing devices $[9,10]$. The test consists of three components: olfactory threshold, olfactory discrimination, and olfactory identification. The total of these three scores gives the overall threshold-discrimination identification (TDI) score. The TDI score is generally considered to be normal at $\geq 30.5$. Subjects with a TDI score of $<$ 30.5 are considered to have olfactory dysfunction.

The primary outcome in this meta-analysis was the mean TDI score. The secondary outcomes were olfactory dysfunction rate, correlations between TDI and AHI as well as TDI and age, and the impact of PAP treatment on TDI.

\section{Statistical analysis}

Meta-analysis was performed using MedCalc version 19.3 (MedCalc Software, Ostend, Belgium). When $I^{2}>50 \%$ and $p<0.05$, heterogeneity was considered statistically significant. The significance of the heterogeneity was used to determine whether the data would be analyzed with a fixed or a 
Fig. 1 Flowchart for selection of studies according to inclusion/ exclusion criteria

Articles identified through database searching
(PubMed/MEDLINE, Google Scholar, SAGE, Cochrane)
$(\mathrm{n}=552)$

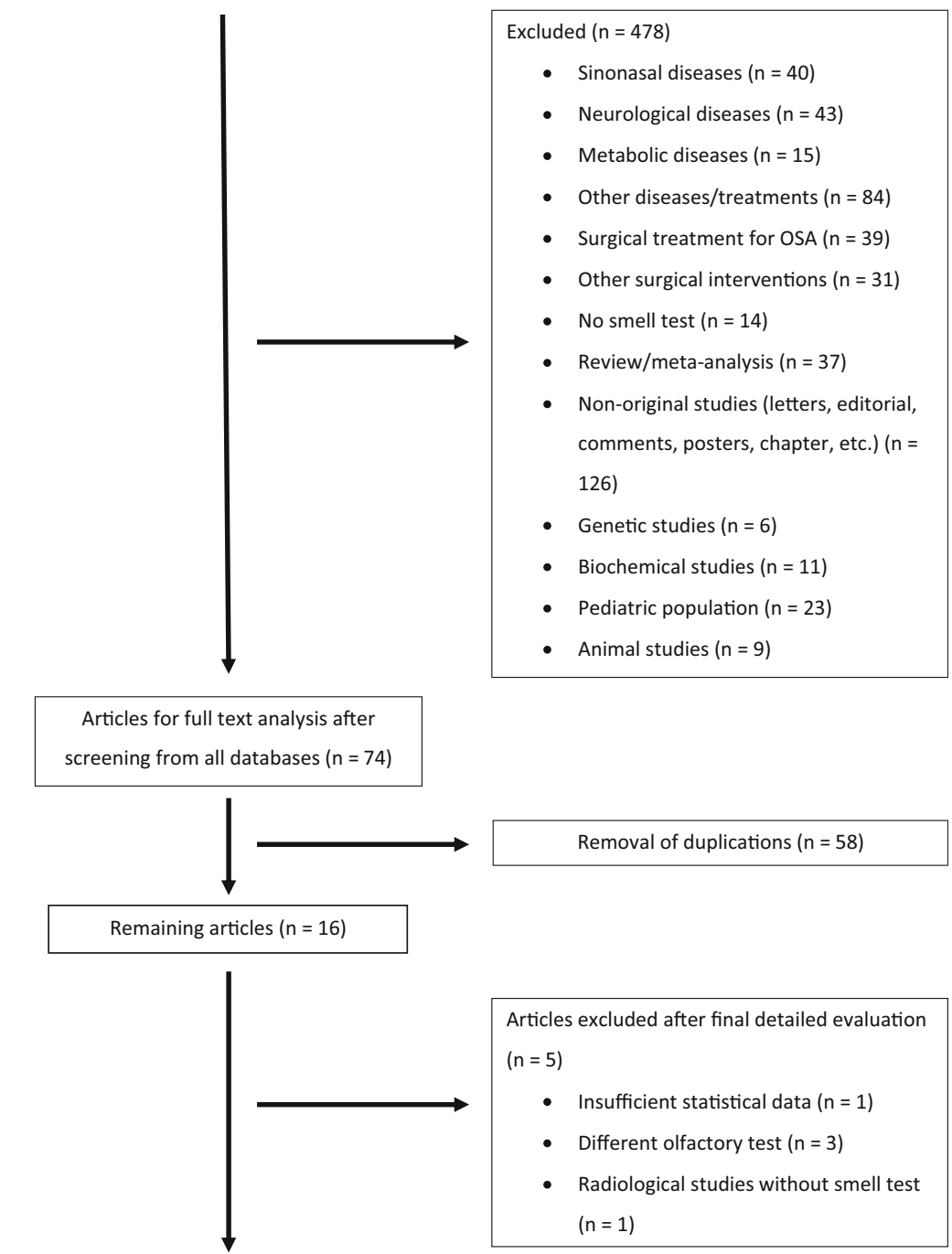

Finally included in the meta-analysis $(n=11)$ random-effects model. The meta-analysis was based on a three-step assessment: (1) The proportional rate of olfactory dysfunction in cases with OSA was determined, (2) the comparison of OF between patients with OSA and the control subjects was performed, and (3) the comparison was made between the TDI scores before and after PAP treatment. Correlations between TDI and age and between TDI and AHI were also evaluated.

\section{Quality assessment of the studies included in this meta-analysis}

The National Institute for Health and Clinical Excellence (NICE) quality assessment tool was used to evaluate the studies included in this review. The following eight items were investigated for each study according to the NICE checklist:

(1) Was the case series collected in more than one center (i.e., multicenter study)?

(2) Is the hypothesis/aim/objective of the study clearly described?

(3) Are the inclusion and exclusion criteria (case definition) clearly reported?

(4) Is there a clear definition of the outcomes reported?

(5) Were data collected prospectively?

(6) Is there an explicit statement that patients were recruited consecutively?

(7) Are the main findings of the study clearly described? 
(8) Are the outcomes stratified (e.g., by abnormal results, disease stage, and patient characteristics)?

The total quality score for each study was calculated.

\section{Results}

The database search identified 552 articles. According to the exclusion criteria, 478 studies were excluded in the first step. After removing duplicates from the remaining 74 studies, 16 studies were found to be relevant. After a detailed evaluation, another 5 studies were excluded; thus, 11 studies involving 557 patients with a diagnosis of OSA were finally included in this meta-analysis (Fig. 1) [6-8, 11-18]. The largest study sample of patients with OSA was enrolled in the study by Liu et al. $(n=80)$, and the lowest number in the study by Versace et al. [8, 17] The gender information revealed the enrolment of 558 males (79\%) and 145 females (21\%), including the control groups. The average age of the patients in the studies ranged from 43.9 to 56.0 years. The mean AHI values were not reported clearly in all the included studies but were mostly given in the part of subgroups according to OSA severity such as mild, moderate, severe, mild to moderate, and moderate to severe (Table 1).

\section{Assessment of the rate of olfactory dysfunction in OSA cases}

Among the studies included in this meta-analysis, only seven studies contain the data of olfactory dysfunction rate in patients with OSA (Table 2). The random-effects model was used to analyze the data (test for heterogeneity; $Q=$ 75.4158, $p<0.0001, I^{2}$ (inconsistency) $=92.04 \%$ ). Of 397 patients with OSA, the general rate of olfactory dysfunction was 73\% (95\% CI: 56.481-87.057) (Fig. 2).

\section{The comparison of olfactory functions between study and control groups}

Of the studies included in this meta-analysis, five case-control studies compared OF between patients with OSA and patients without OSA $(\mathrm{AHI}<5)$ (Table 3). There were 291 patients with OSA, and 252 control subjects (AHI < 5). Some of the studies reported the results in subgroups according to the AHI severity; thus, the subgroups of these studies were evaluated separately in the meta-analysis calculation. The random-effects model was used to analyze the data (test for heterogeneity; $Q=84.1153, p<$ $0.0001, I^{2}$ (inconsistency) $=90.49 \%$ ). The meta-analysis results showed that patients with OSA had lower TDI scores compared to the control subjects and the difference was statistically significant $(p<0.001$, standardized mean difference $(\mathrm{SMD})=-1.831,95 \%$ CI: -2.483 to -1.180 ) (Fig. 3).

\section{Assessment of the effect of PAP treatment on olfactory function}

Four studies with 121 patients investigated the effect of PAP therapy on OF (Table 4). The total duration of PAP therapy was 3 months in three studies, whereas it was 2 months in one study. The fixed-effects model was used to analyze the data (test for heterogeneity; $Q=5.7024, p=0.1270, I^{2}$ (inconsistency) $=47.39 \%$ ). The meta-analysis results showed that PAP treatment significantly improved the TDI scores in patients with OSA $(p<0.001, \mathrm{SMD}=-0.527,95 \% \mathrm{CI}: 0.27-$ 0.78) (Fig. 4).

\section{Assessment of correlations}

Six studies with 354 patients evaluated the correlation between AHI and TDI scores. The random-effects model was used to analyze the data (test for heterogeneity; $Q=31.3286, p$ $<0.0001, I^{2}$ (inconsistency) $=84.04 \%$ ). The meta-analysis results showed a significant negative correlation between the severity of AHI and TDI scores $(p=0.001, z=-3.377, r=$ $-0.438,95 \%$ CI: -0.631 to -0.195$)$.

Three studies with 135 patients evaluated the correlation between the age of the participants and TDI scores. The fixedeffects model was used to analyze the data (test for heterogeneity; $Q=3.8671, p=0.1446, I^{2}$ (inconsistency) $=48.28 \%$ ). The meta-analysis results showed a significant negative correlation between age and TDI scores $(p=0.007, z=-2.695, r$ $=-0.236,95 \% \mathrm{CI}:-0.392$ to -0.0654$)$.

\section{Methodological quality of the included studies}

Of the 11 included studies, 5 were case-control studies, 4 were before and after PAP studies, while 2 were case series. The NICE quality assessment tool demonstrated that the studies met between five and seven points from the total eight points $(7,5,6,6,6,6,6,6,6,6$, and 6 points).

\section{Discussion}

The pathophysiological origin of OSA is the upper airway, which also includes the nasal passages. The peripheral olfactory system consists mainly of the nose, the nasal cavities, and the olfactory epithelium. Despite this close anatomical positional relationship, whether $\mathrm{OF}$ is affected in patients with OSA remains unclear. Furthermore, OSA is known to cause undesired neurocognitive effects, which may also be associated with the central olfactory system. In this meta-analysis, the assessment of OF in patients with OSA was conducted. This topic (i.e., OF in OSA) is still new but has been stagnant for some time. All the studies examined in this meta-analysis were published between 2014 and 2020 . 


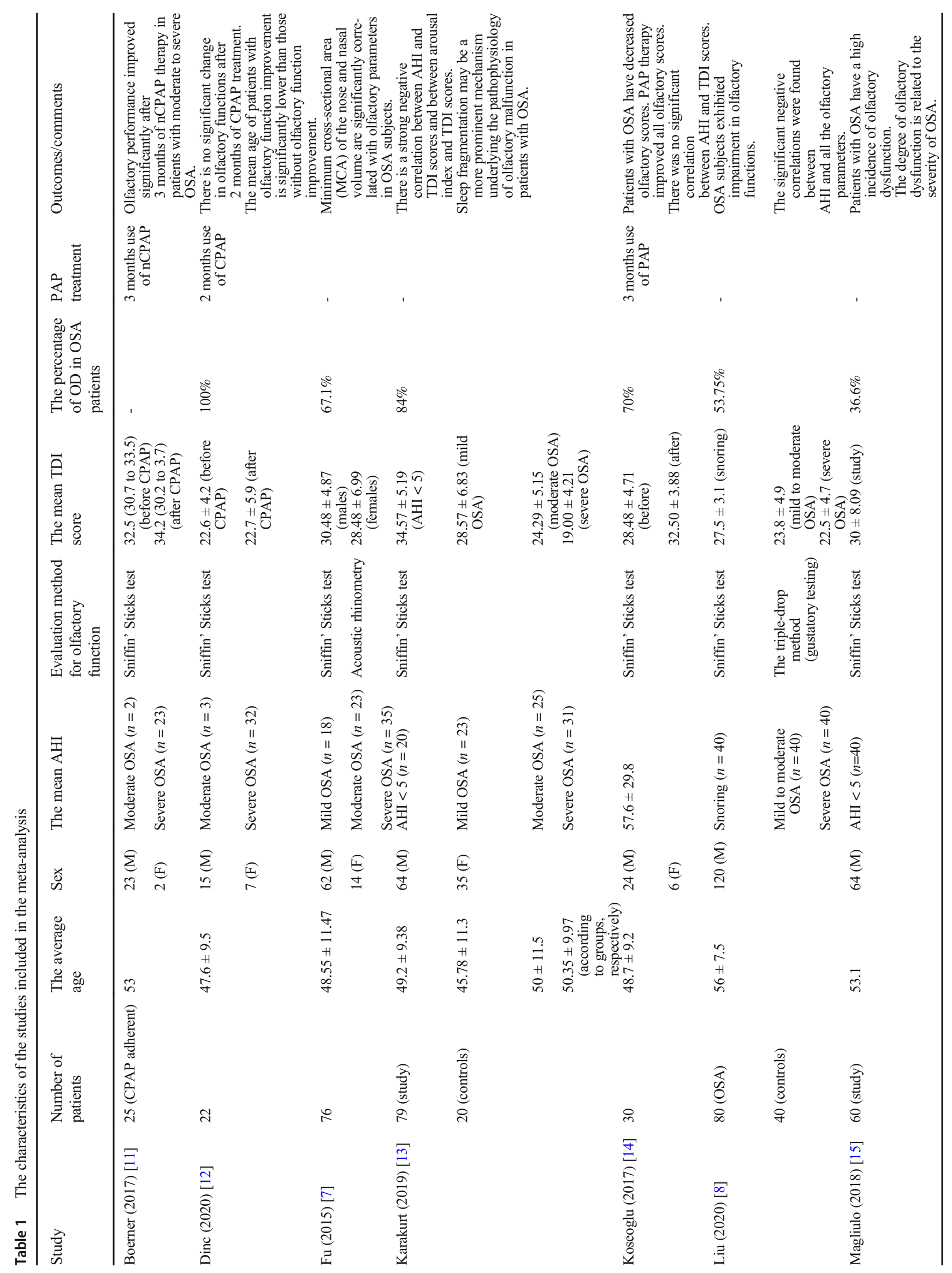




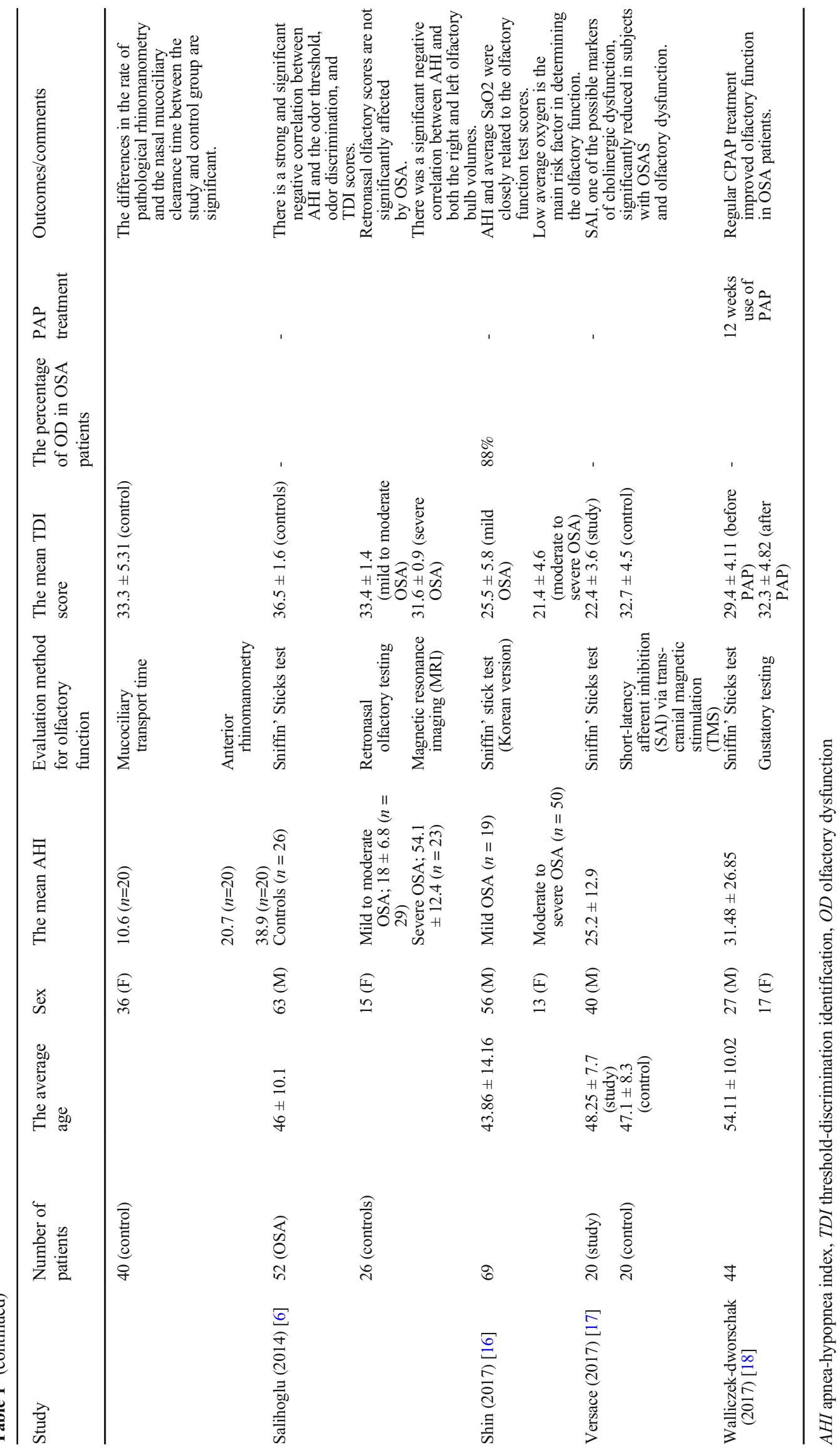


The results of this meta-analysis showed four important outcomes:

(1) Baseline olfactory scores of patients with OSA were lower compared to normal reference levels.

(2) OF of patients with OSA was reduced significantly compared to those without OSA.

(3) The use of PAP therapy provided significant improvement in OF in OSA patients.

(4) OF scores showed significant negative correlations both with age and with the severity of OSA.

Two mechanisms have been primarily suggested to explain the pathophysiology of olfactory dysfunction in OSA. One is the local effect of OSA on the nasal mucosa and local neuropathy because of long-standing vibrations and hypoxia of the nerves. OSA is usually caused by multilevel obstructions, and most of the patients with OSA have concurrent nasal obstruction [19]. As it is the beginning of the upper airway, any resistance in the nasal airway will alter the airflow dynamics. This resistance can interrupt the route of odorants reaching the olfactory region, leading to olfactory dysfunction. Fu et al. investigated the relationship between nasal structure and OF using acoustic rhinometry and the Sniffin' Sticks test in patients with OSA and found that the minimum cross-sectional area of the nose and nasal volume was significantly correlated with olfactory parameters in OSA subjects [7]. Based on these outcomes, they speculated that performing nasal surgery to increase airflow in OSA subjects may also improve olfactory performance. According to Magliulo et al., nasal obstruction directly affects OF in patients with OSA because the impairment resulting from the anterior rhinomanometry was more common among patients with olfactory dysfunction than in those without olfactory dysfunction [15]. In addition, OSA patients usually show poor mucociliary clearance, which leads to disrupted interaction between odorants and the olfactory epithelium $[15,20]$. Further studies are also needed to evaluate whether proinflammatory markers in the nasal airway, which have been previously shown to be increased in the upper airway samples of patients with OSA, contribute to olfactory alterations.

The other mechanism can be explained by the neurocognitive effects of OSA. Previous studies have shown that neurocognitive dysfunction leads to olfactory malfunctioning in patients with neurodegenerative diseases such as Alzheimer's and Parkinson's [21-23]. Similarly, patients with OSA have impaired cognitive functions as a result of sleep fragmentation, sleep loss, arousals, intermittent hypoxia, and hypoxia-reoxygenation episodes [24-26]. It has also been reported that nocturnal hypoxemia causes neuronal loss in the prefrontal, posterior parietal cortex, and hippocampus [27]. Eventually, cognitive deficits in patients with OSA may affect memory, attention, and executive functions. Daurat et al. found that the number of microarousals was the best predictor of memory deficit in patients with OSA [28]. Therefore, they suggested a possible link between episodic memory deficit and sleep-sensitive brain areas, such as the hippocampus, which are also associated with the olfactory pathways. Several studies have revealed that subjects who are successful in the assessment of executive functioning also show good scores in discrimination and identification of more odors [29, 30]. In the most recent study, Liu et al. suggested that olfactory dysfunction in patients with OSA may be caused by impaired cognitive functions that occur in OSA subjects [8].

Beyond these explanations, another possible mechanism is related to the alterations in cholinergic neurotransmission. Versace et al. investigated this issue by evaluating the shortlatency afferent inhibition of the motor cortex in addition to the assessment of OF in patients with OSA [17]. The OSA patients were found to have a significantly reduced shortlatency afferent inhibition supporting cholinergic dysfunction. The occurrence of olfactory dysfunction might indicate a cognitive impairment triggered by the alterations in neurotransmitter balance in patients with OSA. The reason for this situation in OSA is dependent on impaired sleep architecture, sleep fragmentation, intermittent hypoxia, deterioration of REM/non-REM sleep quality, and increased daytime sleepiness [17, 27].

There are various connections and pathways from the olfactory bulb to the brain, such as the piriform cortex, the entorhinal cortex, the orbitofrontal cortex, the anterior olfactory nucleus, the periamygdaloid hippocampal region, and the hypothalamus, as well as tertiary connections to the limbic and autonomic systems in the brain [31]. Previous studies have shown that cell neurogenesis and density are reduced, especially in the hippocampus, the frontal cortex, and generalized gray matter in patients with OSA $[32,33]$. According to these outcomes, it can be hypothesized that the factors causing neurocognitive dysfunction in patients with OSA can lead to olfactory dysfunction in those patients. To the best of our knowledge, no study has demonstrated both neurocognitive dysfunction and olfactory dysfunction at the same time in the same population with OSA. Therefore, there is a need for future research to reveal this relationship using more objective and quantitative methods.

The Sniffin' Sticks test was used as the main method to evaluate OF in this meta-analysis, as most of the studies investigated OF using this test. However, Invitto et al. assessed olfactory perception in patients with OSA through the chemosensory event-related potentials (CSERP) [34]. The CSERP of patients with OSA was found to differ from that of healthy subjects, and OSA subjects showed greater CSERP in the right inferior frontal cortex and the left centroparietal suggesting an impairment in endogenous components. Given these outcomes, based on an objectively quantifiable olfactory 
Table 2 The proportion of olfactory dysfunction and the meta-analysis

\begin{tabular}{|c|c|c|c|c|c|c|}
\hline \multirow[t]{2}{*}{ Study } & \multirow[t]{2}{*}{ Sample size } & \multirow{2}{*}{$\begin{array}{l}\text { Number of cases with } \\
\text { olfactory dysfunction }\end{array}$} & \multirow[t]{2}{*}{ Proportion (\%) } & \multirow[t]{2}{*}{$95 \% \mathrm{CI}$} & \multicolumn{2}{|c|}{ Weight (\%) } \\
\hline & & & & & Fixed & Random \\
\hline Dinc (2020) & 22 & 22 & 100,000 & 84,563 to 100,000 & 5.69 & 12.99 \\
\hline $\mathrm{Fu}(2015)$ & 76 & 51 & 67,105 & 55,374 to 77,457 & 19.06 & 14.80 \\
\hline Karakurt (2019) & 79 & 66 & 83,544 & 73,505 to 90,937 & 19.80 & 14.83 \\
\hline Koseoglu (2017) & 30 & 21 & 70,000 & 50,604 to 85,265 & 7.67 & 13.60 \\
\hline Liu (2020) & 80 & 43 & 53,750 & 42,242 to 64,972 & 20.05 & 14.84 \\
\hline Magliulo (2018) & 60 & 22 & 36,667 & 24,594 to 50,103 & 15.10 & 14.57 \\
\hline Shin (2017) & 50 & 44 & 88,000 & 75,690 to 95,466 & 12.62 & 14.36 \\
\hline Total (fixed effects) & 397 & 269 & 69,380 & 64,632 to 73,842 & 100.00 & 100.00 \\
\hline Total (random effects) & 397 & 269 & 73,194 & 56,481 to 87,057 & 100.00 & 100.00 \\
\hline
\end{tabular}

assessment, that study is important for future research regarding olfactory assessment in patients with OSA.

Recent studies have shown some evidence that the likely effect of OSA on OF is an impairment, which can be reversed by PAP therapy $[11,14,18]$. There are conflicting results from different studies regarding the effect of PAP treatment on the nasal mucosa. On the one hand, PAP can itself induce nasal inflammation due to its "mechanical stimulus" effect on the nasal mucosa [35]. On the other hand, intranasal inflammation can be reportedly reduced by regular PAP use [36]. According to the results of this meta-analysis, the use of PAP therapy for at least 3 months may provide a significant improvement in OF in patients with OSA.
A long-lasting use with good adherence may be even more beneficial to improve $\mathrm{OF}$ in these patients-for example, a cohort study of PAP adherent patients with a follow-up period of at least 1 year may demonstrate more reliable results. Since the studies evaluating the effect of PAP treatment on OF only provided TDI scores, it is not possible to comment on the clinical outcomes of PAP treatment. Only the study by Boerner et al. provided the data of sense of smell self-estimation VAS, which was not significantly changed after 3 months of CPAP [11]. Further studies are needed to investigate if the olfactory dysfunction rate expressed by TDI scores is in line with clinical significance. Another issue is that there are no data of the effect of non-PAP treatment on OF in patients
Fig. 2 The proportional rate of olfactory dysfunction in patients with obstructive sleep apnea and the meta-analysis

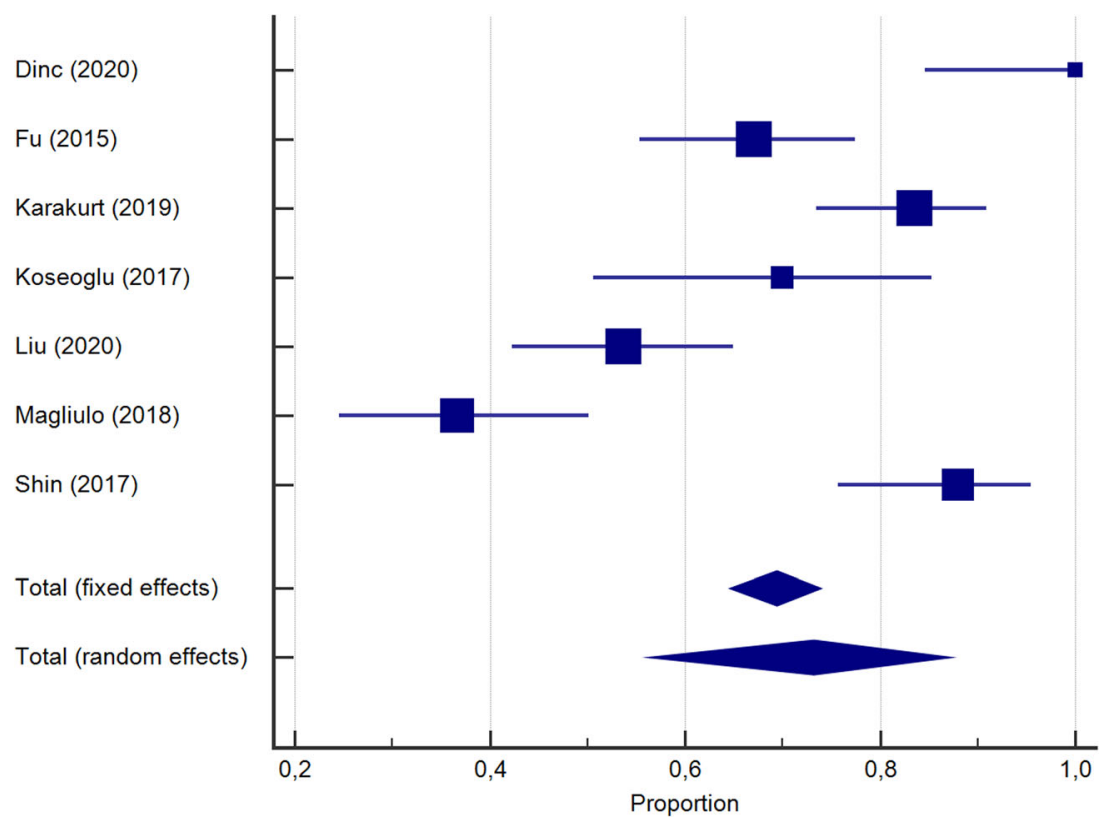


with OSA. It can be speculated that if PAP therapy could become successful in the amelioration of olfactory dysfunction by treating OSA, other alternatives to PAP such as upper airway surgery, oral appliances, and neurostimulation might also be effective if these treatments provide acceptable success in relieving OSA.

There is evidence that the olfactory bulb volume measured on magnetic resonance imaging (MRI) is a good indicator of OF $[37,38]$. In an MRI study by Salihoglu et al., there was found to be a significant negative correlation of AHI with odor identification score and both the right and the left olfactory bulb volumes [6]. Damm et al. used a combined assessment method using both the Sniffin' Sticks test and MRI and found that there are two important areas in the nasal cavity where olfactory performance is the most affected: the segment in the upper meatus below the cribriform plate and the anterior segment of the inferior meatus [39]. The latter finding supports the importance of the inferior turbinate region for OF. Therefore, surgical interventions on inferior turbinate might affect $\mathrm{OF}$.

According to the results of this meta-analysis, the TDI scores showed significant negative correlations both with age and the severity of OSA (AHI). According to Dinc et al., age is an important factor for olfactory functions in patients with OSA, as they obtained a better improvement following a 2-month PAP treatment in younger patients compared to the older patients [12]. OF reportedly tends to deteriorate in the elderly. Similarly, the incidence of OSA increases in elderly patients because of impaired neuromuscular dysfunction. Moreover, the cognitive impairment that comes with aging may also affect odor functions. Despite these age-related confounding factors, none of the OSA groups included in this meta-analysis differed from the control groups in terms of age, but they showed higher rate of olfactory dysfunction than the control subjects. The average age of the patients with OSA ranged from 43.9 to 56.0 years in the included studies, and the overall incidence of olfactory dysfunction in healthy individuals of the same age population is around 20\% [40,41]. According to the results of this meta-analysis, the rate of olfactory dysfunction in patients with OSA is $73 \%$. This outcome should be kept in mind when evaluating patients with OSA.

This study has a few limitations: (1) The total number of included studies was limited, and moreover, metaanalysis calculations for subgroup evaluations had to be performed with even fewer studies. (2) Male predominance $(79 \%)$ was significant in all the included studies, so the results from this meta-analysis mainly indicate the OF of males with OSA. (3) As nasal obstruction is common in OSA subjects, making an olfactory assessment in these patients without excluding all kinds of nasal 
Fig. 3 The meta-analysis based on the comparison of the threshold-discrimination identification scores between the patients and controls (expressed as standardized mean difference)

Karakurt (2019) (mild OSA)
Karakurt (2019) (moderate OSA)
Karakurt (2019) (severe OSA)
Liu (2020) (mild to moderate OSA)
Liu (2020) (severe OSA)
Magliulo (2018)
Salihoglu (2014) (mild to moderate OSA)
Salihoglu (2014) (severe OSA)
Versace (2017)
Total (fixed effects)
Total (random effects)

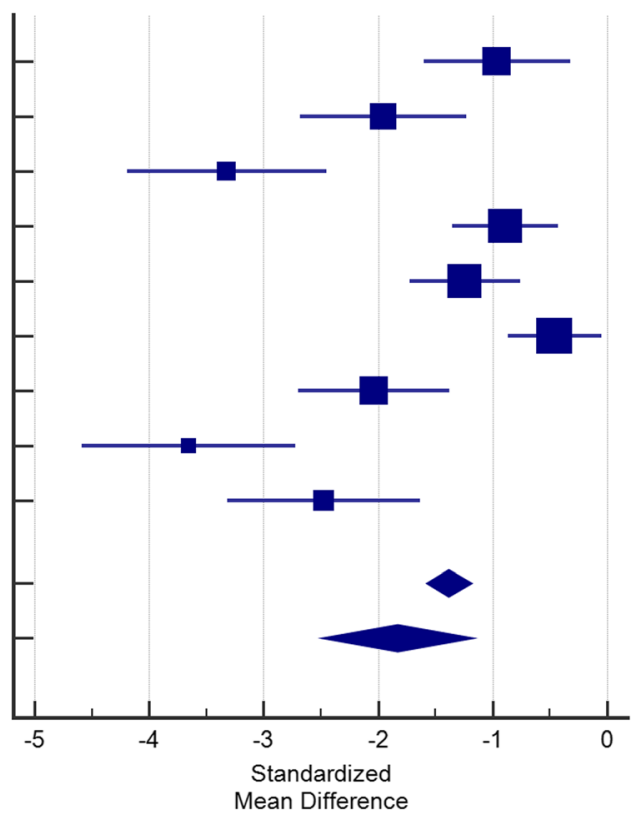

abnormalities will cause controversial outcomes. This subject is evaluated based on the examination findings given by the authors of the included articles. This methodological limitation can only be overcome by using additional methods such as complete radiological assessment of the patients. (4) Since the mean NICE quality score of the included studies was six points out of eight in total, it must be emphasized that future study designs on this subject should be further developed. The readers should keep these points in mind regarding the generalizability of this meta-analysis on the OSA population.

\section{Conclusion}

This meta-analysis showed that olfactory performance is reduced in patients with OSA. However, this impairment seems to be reversible when PAP treatment is effectively used. OF may worsen with the increasing severity of OSA and aging. To understand and clarify the exact pathophysiology of olfactory dysfunction in OSA, there is a need for further research using more objective and quantitative assessments, and combining radiological, neurological, and physiological methods.

Table 4 The studies giving the outcomes of PAP treatment and the meta-analysis

\begin{tabular}{|c|c|c|c|c|c|c|c|c|c|c|c|}
\hline \multirow[t]{2}{*}{ Study } & \multirow[t]{2}{*}{ Sample } & \multirow{2}{*}{$\begin{array}{l}\text { The mean } \\
\text { TDI before } \\
\text { PAP }\end{array}$} & \multirow[t]{2}{*}{$\mathrm{SD}$} & \multirow{2}{*}{$\begin{array}{l}\text { The mean } \\
\text { TDI after PAP }\end{array}$} & \multirow[t]{2}{*}{$\mathrm{SD}$} & \multirow[t]{2}{*}{ SMD } & \multirow[t]{2}{*}{$95 \% \mathrm{CI}$} & \multirow[t]{2}{*}{$t$} & \multirow[t]{2}{*}{$p$} & \multicolumn{2}{|c|}{ Weight $(\%)$} \\
\hline & & & & & & & & & & Fixed & Random \\
\hline Boerner (2017) & 25 & 32.5 & 4.34 & 34.2 & 4.86 & -0.363 & -0.928 to 0.201 & & & 21.41 & 23.35 \\
\hline Dinc (2020) & 22 & 22.6 & 4.2 & 22.7 & 5.9 & -0.0192 & -0.617 to 0.578 & & & 19.25 & 21.97 \\
\hline Koseoglu (2017) & 30 & 28.48 & 4.71 & 32.5 & 3.88 & -0.920 & -1.457 to -0.382 & & & 23.44 & 24.54 \\
\hline Walliczek-dworschak (2017) & 44 & 29.4 & 4.11 & 32.3 & 4.82 & -0.642 & -1.073 to -0.211 & & & 35.90 & 30.15 \\
\hline Total (fixed effects) & 121 & & & & & -0.527 & -0.783 to -0.272 & -4.060 & $<0.001$ & 100.00 & 100.00 \\
\hline Total (random effects) & 121 & & & & & -0.508 & -0.866 to -0.151 & -2.800 & 0.006 & 100.00 & 100.00 \\
\hline
\end{tabular}

$P A P$ positive airway pressure, $T D I$ threshold-discrimination identification score, $S D$ standard deviation, $S M D$ standardized mean difference 
Fig. 4 The meta-analysis showing the effect of PAP treatment

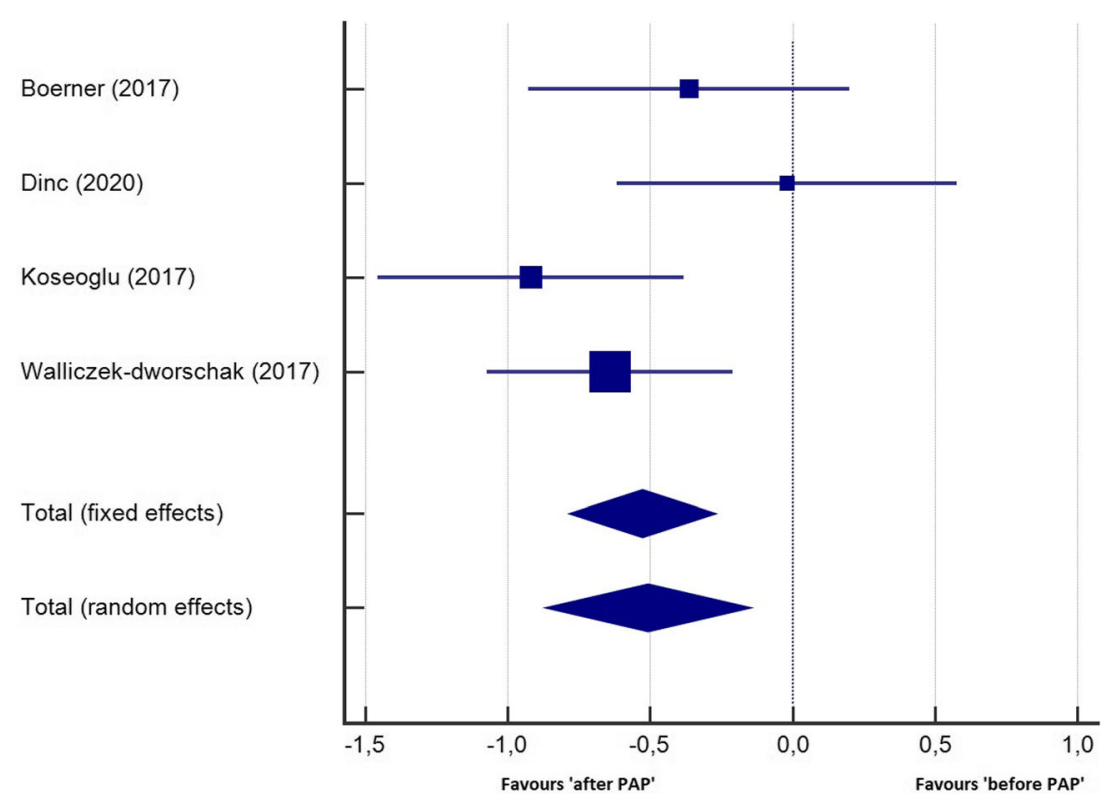

van den Brink M, Schmuker M, Fischmeister FPS, Heinbockel T, Shields VDC, Faraji F, Santamaría E, Fredborg WEA, Morini G, Olofsson JK, Jalessi M, Karni N, D'Errico A, Alizadeh R, Pellegrino R, Meyer P, Huart C, Chen B, Soler GM, Alwashahi MK, Welge-Lüssen A, Freiherr J, de Groot JHB, Klein H, Okamoto M, Singh PB, Hsieh JW, GCCR Group Author, Abdulrahman O, Dalton P, Yan CH, Voznessenskaya VV, Chen J, Sell EA, WalshMessinger J, Archer NS, Koyama S, Deary V, Roberts SC, Yanık H, Albayrak S, Nováková LM, Croijmans I, Mazal PP, Moein ST, Margulis E, Mignot C, Mariño S, Georgiev D, Kaushik PK, Malnic B, Wang H, Seyed-Allaei S, Yoluk N, Razzaghi-Asl S, Justice JM, Restrepo D, Abdulrahman O, Dalton P, Yan CH, Voznessenskaya VV, Chen J, Sell EA, Walsh-Messinger J, Archer NS, Koyama S, Deary V, Roberts SC, Yanık H, Albayrak S, Nováková LM, Croijmans I, Mazal PP, Moein ST, Margulis E, Mignot C, Mariño S, Georgiev D, Kaushik PK, Malnic B, Wang H, Seyed-Allaei S, Yoluk N, Razzaghi-Asl S, Justice JM, Restrepo D, Reed DR, Hummel T, Munger SD, Hayes JE (2020) More than smellCOVID-19 is associated with severe impairment of smell, taste, and chemesthesis. Chem Senses 45(7):609-622

6. Salihoğlu M, Kendirli MT, Altundağ A, Tekeli H, Sağlam M, Çayönü M, Șenol MG, Özdağ F (2014) The effect of obstructive sleep apnea on olfactory functions. Laryngoscope 124(9):2190 2194

7. Fu D, Pinto JM, Wang L, Chen G, Zhan X, Wei Y (2015) The effect of nasal structure on olfactory function in patients with OSA. Eur Arch Otorhinolaryngol 272(2):357-362

8. Liu Y, Fang F, Zhan X, Yao L, Wei Y (2020) The impact of obstructive apnea sleep syndrome on chemical function. Sleep Breath [published online ahead of print]

9. Kobal G, Hummel T, Sekinger B, Barz S, Roscher S, Wolf S (1996) "Sniffin' Sticks": screening of olfactory performance. Rhinology 34(4):222-226

10. Wolfensberger M, Schnieper I, Welge-Lüssen A (2000) Sniffin' Sticks: a new olfactory test battery. Acta Otolaryngol 120(2):303306 
11. Boerner B, Tini GM, Fachinger P, Graber SM, Irani S (2017) Significant improvement of olfactory performance in sleep apnea patients after three months of nasal CPAP therapy-observational study and randomized trial. PLoS One 12(2):e0171087

12. Dinc AS, Cayonu M, Duyar SS, Firat S (2020) Increased age adversely affects the therapeutic effect of CPAP treatment for olfactory functions. Sleep Breath 24(1):83-88

13. Karakurt SE, Karakuș MF, Çolak M, Akbal Ș, Çetin MA, İkincioğulları A, Dere HH (2020) Evaluation of olfactory function in patients with obstructive sleep apnea syndrome. Sleep Breath 24(3):1137-1142

14. Koseoğlu S, Derin S, Yilmaz M, Kutlu G, Sahan M (2017) Does positive airway pressure therapy improve olfactory function? Int Forum Allergy Rhinol 7(6):557-560

15. Magliulo G, De Vincentiis M, Iannella G, Ciofalo A, Pasquariello B, Manno A et al (2018) Olfactory evaluation in obstructive sleep apnoea patients. Acta Otorhinolaryngol Ital 38(4):338-345

16. Shin DH, Ahn SH, Yang Y, Choi S, Cho JH, Hong SC, Kim JK (2017) The effect of sleep disordered breathing on olfactory functions: analysis by apnea-hypopnea index. Clin Exp Otorhinolaryngol 10(1):71-76

17. Versace V, Langthaler PB, Sebastianelli L, Golaszewski S, Kunz AB, Brigo F, Saltuari L, Nardone R (2017) Cholinergic neurotransmission and olfactory function in obstructive sleep apnea syndrome: a TMS study. Sleep Med 37:113-118

18. Walliczek-Dworschak U, Cassel W, Mittendorf L, Pellegrino R, Koehler U, Güldner C, Dworschak POG, Hildebrandt O, Daniel H, Günzel T, Teymoortash A, Hummel T (2017) Continuous positive air pressure improves orthonasal olfactory function of patients with obstructive sleep apnea. Sleep Med 34:24-29

19. Krakow B, Foley-Shea M, Ulibarri VA, McIver ND, Honsinger R (2016) Prevalence of potential nonallergic rhinitis at a communitybased sleep medical center. Sleep Breath 20(3):987-993

20. Deniz M, Gultekin E, Ciftci Z, Alp R, Ozdemir DN, Isik A, Demirel OB (2014) Nasal mucociliary clearance in obstructive sleep apnea syndrome patients. Am J Rhinol Allergy 28(5):178-180

21. Ubeda-Bañon I, Saiz-Sanchez D, Flores-Cuadrado A, RiojaCorroto E, Gonzalez-Rodriguez M, Villar-Conde S, AstilleroLopez V, Cabello-de la Rosa JP, Gallardo-Alcañiz MJ, Vaamonde-Gamo J, Relea-Calatayud F, Gonzalez-Lopez L, Mohedano-Moriano A, Rabano A, Martinez-Marcos A (2020) The human olfactory system in two proteinopathies: Alzheimer's and Parkinson's diseases. Transl Neurodegener 9(1):22

22. Dibattista M, Pifferi S, Menini A, Reisert J (2020) Alzheimer's disease: what can we learn from the peripheral olfactory system? Front Neurosci 14:440

23. Doty RL (2012) Olfactory dysfunction in Parkinson disease. Nat Rev Neurol 8(6):329-339

24. Rosenzweig I, Glasser M, Polsek D, Leschziner GD, Williams SC, Morrell MJ (2015) Sleep apnoea and the brain: a complex relationship. Lancet Respir Med 5:404-414

25. Lal C, Strange C, Bachman D (2012) Neurocognitive impairment in obstructive sleep apnea. Chest 141(6):1601-1610

26. Verstraeten E (2007) Neurocognitive effects of obstructive sleep apnea syndrome. Curr Neurol Neurosci Rep 7(2):161-166

27. Barnes DC, Wilson DA (2014) Sleep and olfactory cortical plasticity. Front Behav Neurosci 8:134

28. Daurat A, Foret J, Bret-Dibat JL, Fureix C, Tiberge M (2008) Spatial and temporal memories are affected by sleep fragmentation in obstructive sleep apnea syndrome. J Clin Exp Neuropsychol 30(1):91-101

29. Dulay MF, Gesteland RC, Shear PK, Ritchey PN, Frank RA (2008) Assessment of the influence of cognition and cognitive processing speed on three tests of olfaction. J Clin Exp Neuropsychol 30(3): 327-337

30. Hedner M, Larsson M, Arnold N, Zucco GM, Hummel T (2010) Cognitive factors in odor detection, odor discrimination, and odor identification tasks. J Clin Exp Neuropsychol 32(10):1062-1067

31. Parent A, Carpenter MB (1997) Carpenter's human neuroanatomy, vol 19. Williams \& Wilkins, Baltimore

32. Torelli F, Moscufo N, Garreffa G, Placidi F, Romigi A, Zannino S, Bozzali M, Fasano F, Giulietti G, Djonlagic I, Malhotra A, Marciani MG, Guttmann CRG (2011) Cognitive profile and brain morphological changes in obstructive sleep apnea. Neuroimage 54(2):787-793

33. Zimmerman ME, Aloia MS (2006) A review of neuroimaging in obstructive sleep apnea. J Clin Sleep Med 2(4):461-471

34. Invitto $\mathrm{S}$, Calcagnì $\mathrm{A}$, Piraino $\mathrm{G}$, Ciccarese $\mathrm{V}$, Balconi $\mathrm{M}$, De Tommaso $\mathrm{M}$ et al (2019) Obstructive sleep apnea syndrome and olfactory perception: an OERP study. Respir Physiol Neurobiol 259:37-44

35. Almendros I, Acerbi I, Vilaseca I, Montserrat JM, Navajas D, Farré $\mathrm{R}$ (2008) Continuous positive airway pressure (CPAP) induces early nasal inflammation. Sleep 31(1):127-131

36. Gelardi M, Carbonara G, Maffezzoni E, Marvisi M, Quaranta N, Ferri R (2012) Regular CPAP utilization reduces nasal inflammation assessed by nasal cytology in obstructive sleep apnea syndrome. Sleep Med 13(7):859-863

37. Hummel T, Smitka M, Puschmann S, Gerber JC, Schaal B, Buschhüter D (2011) Correlation between olfactory bulb volume and olfactory function in children and adolescents. Exp Brain Res 214(2):285-291

38. Haehner A, Rodewald A, Gerber JC, Hummel T (2008) Correlation of olfactory function with changes in the volume of the human olfactory bulb. Arch Otolaryngol Head Neck Surg 134(6):621-624

39. Damm M, Vent J, Schmidt M, Theissen P, Eckel HE, Lötsch J, Hummel T (2002) Intranasal volume and olfactory function. Chem Senses 27(9):831-839

40. Hummel T, Kobal G, Gudziol H, Mackay-Sim A (2007) Normative data for the "Sniffin' Sticks" including tests of odor identification, odor discrimination, and olfactory thresholds: an upgrade based on a group of more than 3,000 subjects. Eur Arch Otorhinolaryngol 264(3):237-243

41. Landis BN, Konnerth CG, Hummel T (2004) A study on the frequency of olfactory dysfunction. Laryngoscope 114(10):17641769

Comment In this well written and succinct systemic review and metaanalysis of the literature, the authors show that untreated OSA adversely olfactory function. This effect of OSA is reversible by treatment. Another health and well-being benefit of treating OSA. The pathophysiology of this can't be inferred from this study but it does make a compelling case for further research in OSA.

Ian Wilcox,

NSW, Australia

Publisher's note Springer Nature remains neutral with regard to jurisdictional claims in published maps and institutional affiliations. 